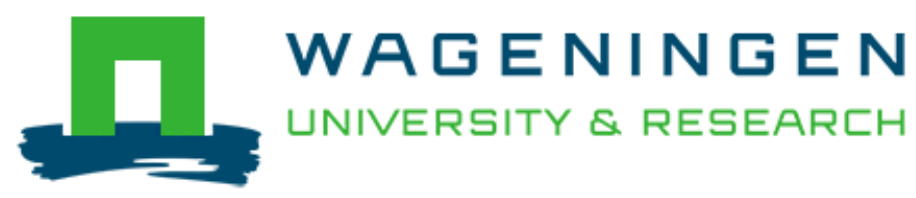

Pepsin diffusivity in whey protein gels and its effect on gastric digestion

Luo, Q., Borst, J. W., Westphal, A. H., Boom, R. M., \& Janssen, A. E. M.

This is a "Post-Print" accepted manuscript, which has been published in "Food Hydrocolloids"

This version is distributed under a non-commercial no derivatives Creative Commons (c) (1) $\Theta \Theta$

(CC-BY-NC-ND) user license, which permits use, distribution, and reproduction in any medium, provided the original work is properly cited and not used for commercial purposes. Further, the restriction applies that if you remix, transform, or build upon the material, you may not distribute the modified material.

Please cite this publication as follows:

Luo, Q., Borst, J. W., Westphal, A. H., Boom, R. M., \& Janssen, A. E. M. (2017). Pepsin diffusivity in whey protein gels and its effect on gastric digestion. Food Hydrocolloids, 66, 318-325. DOI: 10.1016/j.foodhyd.2016.11.046

You can download the published version at:

https://doi.org/10.1016/j.foodhyd.2016.11.046 


\title{
Pepsin diffusivity in whey protein gels and its effect on gastric digestion
}

\author{
Qi Luo ${ }^{\mathrm{a}}$, Jan Willem Borst ${ }^{\mathrm{b}, \mathrm{c}}$, Adrie H. Westphal ${ }^{\mathrm{b}, \mathrm{c}}$, Remko M. Boom ${ }^{\mathrm{a}}$, Anja E.M. Janssen ${ }^{\mathrm{a}, *}$ \\ ${ }^{a}$ Food Process Engineering, Wageningen University, P.O. Box 17, 6700 AA, Wageningen, The Netherlands \\ ${ }^{b}$ Laboratory of Biochemistry, Wageningen University, P.O. Box 8128, 6700 ET, Wageningen, The Netherlands \\ ${ }^{c}$ MicroSpectroscopy Centre Wageningen, Wageningen University, P.O. Box 81286700 ET, Wageningen, The Netherlands
}

\begin{abstract}
Protein is essential to human health, but its digestion kinetics in varied structures are not yet well understood. We previously found different kinetics of protein hydrolysis in solution and in gels, and we hypothesized that the difference stemmed from the steric hindrance of gel structure to the diffusion of pepsin and its hydrolysates. To better understand the pepsin diffusivity in food matrices and its effect on digestion, we determined the diffusivity of pepsin in water and in whey protein isolate (WPI) gels by fluorescence correlation spectroscopy (FCS).We estimated the pepsin concentration gradient during digestion based on the determined diffusivity, which showed that the pepsin is constrained within a thin layer from the gel surface. Gel composition analysis confirmed this constraint: peptides as protein fragments were observed only in the first $2 \mathrm{~mm}$ of the WPI gels after $6 \mathrm{~h}$ of in vitro gastric digestion. Scanning electron microscopy indicated that pepsin loosened the microstructure of whey protein gel surfaces, which may accelerate pepsin diffusion and consequently gel surface disintegration. We conclude that the mode of whey protein gel digestion is determined by the summed effect of diffusion limitation, hydrolysis rate and microstructure transformation.
\end{abstract}

Keywords: Pepsin, GFP, FCS, Diffusion, Whey protein gel, Gastric digestion,

\section{Introduction}

Proteins in foods supply most of the amino acids for protein synthesis in human bodies (Whitney et al., 1998). The digestion of protein starts in the stomach by the acidic environment and pepsin-catalyzed hydrolysis (Singh et al., 2015). Pepsin is an aspartic protease with two aspartic acid residues at the catalytic site (Fersht, 1985) and has maximum activity between pH 1.5 and 2.5 (Piper \& Fenton, 1965). It prefers to cleave a peptide sequence containing phenylalanine, tyrosine, tryptophan and leucine residues (Inouye \& Fruton, 1967).

\footnotetext{
thublished on Food Hydrocolloids by Elsevier, available online at DOI:10.1016/j.foodhyd.2016.11.046. This is an author version typeset by Qi Luo.

${ }^{*}$ Corresponding author

Email address: an ja. janssen@wur.nl (Anja E.M. Janssen) 
Food structure is important in the digestion and nutrient delivery (Bornhorst \& Singh, 2014; Singh et al., 2015); hence, the protein digestion differs in foods with different structures. For example, NyembDiop et al. (2016) investigated the in vitro digestion of four different egg white gel matrices, and found that the gel structure greatly influences the digestion kinetics, resulting in different peptide profiles. (Guo et al., 2015) found varied disintegration kinetics for two differently structured whey protein emulsion gels: the soft gel (hardness: 19.2 N) disintegrated faster, when both fragmentation and abrasion enacted in the disintegration; the hard gel (hardness $69.9 \mathrm{~N}$ ) disintegrated slower, and abrasion was the predominant mechanism. Moreover, the abrasion process was accelerated by the presence of pepsin.

To better understand the role of pepsin in the digestion of protein in food structure, we previously studied the in vitro digestion of protein and protein gels by analyzing the peptide distribution after hydrolysis (Luo et al., 2015). We found that the kinetics of protein hydrolysis in solution and in gels are different: while the dissolved proteins were hydrolyzed through a zipper-type mechanism, the gels followed a slower one-by-one mechanism. We hypothesized that pepsin can penetrate the gel microstructure and hydrolyze proteins in gel matrices. Digestion kinetics may be limited by diffusion of pepsin in gel matrices, which can explain the differences in hydrolysis kinetics.

There are several methods to study the diffusivity of macromolecules in gel networks. Pulsed field gradient NMR has been used to study the diffusion of poly(ethylene glycol)s in whey protein solutions and gels (Colsenet et al., 2006b) and to study the self-diffusion of native proteins and dextran during the heatinduced gelation of $\beta$-lactoglo-bulin (Croguennoc et al., 2001). Fluorescence recovery after photobleaching (FRAP) and fluorescence correlation spectroscopy (FCS) have been used to characterize the diffusions of pectin methylesterases in pectin solutions and gels (Videcoq et al., 2013).

FCS is based on the analysis of intensity fluctuations from some dynamic process, e.g. translational diffusion of a fluorophore through a small volume, using a confocal laser microscope. By autocorrelation analysis of the time-dependent fluorescence fluctuation, the diffusion coefficient of the fluorophore can be determined (Lakowicz, 2006). While pulsed field gradient NMR requires a probe that is distinct from native proteins in the gel network (Croguennoc et al., 2001), the two fluorescence methods (FRAP and FCS) can measure any desired molecules that are fluorescent or fluorescently labeled. Compared with FRAP, FCS has a larger detection range of diffusion time ( $\mu s-s)$, can be used at low fluorophore concentrations and can distinguish multicomponent diffusion (Kwaaitaal et al., 2011). In this study, we considered the possibility of interaction between pepsin and protein gel. Therefore, FCS was employed to investigate the diffusivity of pepsin in the protein gels.

In the FCS experiments, we used whey protein isolate (WPI) gel as a model for a protein-based food matrix. In addition to fluorescently labeled pepsin, enhanced green fluorescent protein (EGFP) was used as a reference molecule due to its intrinsic fluorescence and similar size to pepsin. EGFP is a variant of the wild-type green fluorescent protein, which has increased brightness and folding efficiency (Royant \& 
Noirclerc-Savoye, 2011). By combining the information from the diffusion study with that from gel composition analysis and scanning electron microscopy, we can better understand the role of pepsin diffusion in the digestive process, and thus better understand the role of the food microstructure in digestion.

\section{Material and methods}

\subsection{Material}

WPI (Bipro, lot no. JE 034-70-440-6) was purchased from Davisco Food International, Inc. (Le Sueur, MN, USA). This batch of WPI was reported to have a protein content of $97.9 \mathrm{~g} / 100 \mathrm{~g}$ dry solid. Milli-Q water (resistivity $18.2 \mathrm{M} \Omega \mathrm{cm}$ at $25^{\circ} \mathrm{C}$; Merck Millipore, Billerica, MA, USA) was used in all experiments. The fluorescent dye Alexa Fluor ${ }^{\circledR} 633$ succinimidyl ester (Alexa 633, MW $\approx 1200 \mathrm{Da}$ ) was purchased from Life Technologies (Carlsbad, CA, USA); pepsin from porcine gastric mucosa (lyophilized powder, 3,2004,500 units $/ \mathrm{mg}$ protein, $\mathrm{MW}=34.6 \mathrm{kDa}$ ) and all other chemicals used were purchased from Sigma Aldrich (St. Louis, MO, USA).

EGFP $(\mathrm{MW}=26.9 \mathrm{kDa})$ was produced in-house at Wageningen University, via the method described by (Nolles et al., 2015).

\subsection{Preparation of whey protein isolate gels and simulated gastric fluid}

WPI was dissolved in water (15 wt $\%$ or $20 \mathrm{wt} \%)$ and stirred at room temperature for at least $2 \mathrm{~h}$. The solutions were centrifuged at $1000 \mathrm{rpm}(\sim 200 \mathrm{G}$ relative centrifuge force) for $10 \mathrm{~min}$ to remove large aggregates and air bubbles.

FCS was performed on WPI gels that were prepared as follows: $200 \mu \mathrm{s}$ of the WPI solution was pipetted into each well of an eight-well Nunc Lab-Tek Chambered Coverglass (Thermo Fisher Scientific, Waltham, MA, USA). The system was sealed and heated at $90^{\circ} \mathrm{C}$ for $30 \mathrm{~min}$ in a hot-air oven. The WPI gel was then cooled to room temperature and stored at $4{ }^{\circ} \mathrm{C}$. The thickness of the gel layer was 1-2 mm. Further FCS experiments were performed within 2-3 days.

WPI gels for digestion experiments were prepared in Teflon tubes incubated in a $90{ }^{\circ} \mathrm{C}$ water bath for 30 min, as described previously (Luo et al., 2015). To analyse the texture of the WPI gels, cylindrical samples (diameter $2 \mathrm{~cm}$ and height $2 \mathrm{~cm}$ ) were compressed between two flat plates using a Instron texture analyser 5564 (Instron, Norwood, MA, USA) with test speed of $5 \mathrm{~mm} / \mathrm{min}$. The Young's moduli for WPI 15\% and $20 \%$ were $(18 \pm 6) \mathrm{kPa}$ and $(270 \pm 80) \mathrm{kPa}$ respectively (mean \pm standard deviation of four experiments), calculated from the slope of the stress-strain curve in linear region.

The simulated gastric fluid (SGF) for digestion experiments comprised of pepsin $\left(1 \mathrm{~g} \mathrm{~L}^{-1}\right)$, mucin $\left(1.5 \mathrm{~g} \mathrm{~L}^{-1}\right)$ and $\mathrm{NaCl}\left(8.775 \mathrm{~g} \mathrm{~L}^{-1}\right)$ and was adjusted to $\mathrm{pH}$ 2.0, also as prepared previously (Luo et al., 2015). 


\subsection{Pepsin labeling}

Ten milligrams of pepsin was dissolved in $1 \mathrm{~mL}$ 2-(N-morpholino) ethanesulfonic acid buffer (MES buffer, $10 \mathrm{mmol} \mathrm{L}^{-1}, \mathrm{pH}$ 6.5). The fluorescent dye Alexa 633 was dissolved in dimethyl sulfoxide (DMSO) at $10 \mathrm{mg} \mathrm{mL}^{-1}$. A total amount of $100 \mu \mathrm{L}$ Alexa 633 solution was slowly added in small quantities to the pepsin solution while mixing vigorously. The mixture was incubated at room temperature for $1 \mathrm{~h}$. After incubation, the conjugate was purified by a $5 \mathrm{~mL}$ HiTrap desalting column packed with Sephadex G-25 Superfine medium (GE Healthcare Life Sciences, Little Chalfont, UK). The column was operated with a 2 $\mathrm{mL}$ syringe and the purification process was repeated twice. The purity was verified on an KTA system (GE Healthcare Life Sciences) with dual-wavelength detector; it showed a single peak at the same elution volume at $621 \mathrm{~nm}$ and $280 \mathrm{~nm}$. The conjugate was finally preserved in buffer containing $20 \%$ glycerol at $-25{ }^{\circ} \mathrm{C}$. The final concentration of pepsin was approximately $4 \mathrm{mg} \mathrm{mL}^{-1}$ due to the dilution from the purification step and glycerol.

\subsection{Fluorescence correlation spectroscopy}

Five $\mu \mathrm{L}$ of purified Pepsin-Alexa 633 conjugate (later referred to as PepA633) solution or EGFP was pipetted onto each well of an eight-well plate containing WPI gel. The samples were kept at $4{ }^{\circ} \mathrm{C}$ for approximately $20 \mathrm{~h}$ before measuring. This duration allowed adequate fluorescent molecules to migrate from the surface of the gel toward the glass bottom and be measured. FCS was performed on a Leica TCS SP5X system (Leica Microsystem, Wetzlar, Germany) equipped with a $63 \times 1.20$ NA water immersion objective and a supercontinuum white light laser, which emits a continuous spectrum from 470 to 670 nm. Alexa Fluor 633 was excited by selecting the $621 \mathrm{~nm}$ laser line at a pulsed frequency of $80 \mathrm{MHz}$. Fluorescence was recorded through a size-adjustable pinhole set at $90 \mu \mathrm{m}$, and filtered using a $630 \mathrm{~nm}$ to $690 \mathrm{~nm}$ spectral filter. EGFP was excited at wavelength $488 \mathrm{~nm}$ at the same pulsed frequency. The pinhole was now $75 \mu \mathrm{m}$ and the spectral filter was set at $495 \mathrm{~nm}$ to $525 \mathrm{~nm}$. Fluorescence was recorded via the internal hybrid detector, which was coupled to a PicoHarp 300 TCSPC module (PicoQuant GmbH, Berlin, Germany). For each sample, 30s measurements were repeated 10 times. All individual experiments were performed more than three times at $20^{\circ} \mathrm{C}$ and neutral $\mathrm{pH}$, due to the $\mathrm{pH}$ dependence of the fluorophores. The principles of FCS have been described previously (Lakowicz, 2006; Nolles et al., 2015). Briefly, the obtained fluorescence intensity fluctuation from a confocal volume can be described by an autocorrelation function:

$$
G(t)=\frac{\langle I(t) \times I(t+\tau)\rangle}{\langle I\rangle^{2}}
$$

where $I(t)$ and $I(t+\tau)$ are the fluorescence intensities at timet and $t+\tau ; I$ is the average fluorescence intensity. The fluctuations in intensity are caused by diffusion, and in some cases they are additionally caused by triplet dynamics of the fluorophore. The equation used to fit the translational diffusion data and 
includes triplet state is as follows:

$$
G(\tau)=1+\frac{1}{\langle N\rangle}\left(1+\frac{F_{\text {trip }}}{1-F_{\text {trip }}} \cdot e^{-t / T_{\text {trip }}}\right) \cdot \sum_{i=1}^{n} \frac{F_{i}}{\left(1+\left(t / \tau_{\text {dif }, i}\right)\right) \cdot \sqrt{1+\left(\omega_{x y} / \omega_{z}^{2} \cdot\left(t / \tau_{\text {dif }, i}\right)\right.}}
$$

where $\mathrm{N} ¥$ is the average number of fluorescent particles $\mathrm{N}$ in the spot. The exponential term describes the triplet state behavior, in which Ftrip is the fraction of molecules in the triplet state and Ttrip (ms) is the average time a molecule resides in the triplet state. The last part of the equation describes the diffusion behavior of the molecules, where $\mathrm{n}$ is the number of species, $F_{i}$ is the fraction of species $\mathrm{i}, \tau_{d i f, i}(\mathrm{~ms})$ is the diffusion time of species $\mathrm{i}$ and $\mathrm{xy}(\mathrm{nm})$ and $\mathrm{z}(\mathrm{nm})$ are the equatorial and axial radii of the detection volume, respectively. The diffusion constant Di of species i correlates the observed diffusion time $\tau_{d i f, i}$, according to the following equation:

$$
D_{i}=\frac{\omega_{x y}^{2}}{4 \cdot \tau_{d i f, i}}
$$

The value of $\omega_{x y}$ can be obtained by calibration with a solution of a fluorophore with a known diffusion constant. Alexa $633\left(\mathrm{D}=3.0 \times 10^{-10} \mathrm{~m}^{2} \mathrm{~s}^{-1}\right.$ in water at $20^{\circ} \mathrm{C}$; data from PicoQuant) was used to calibrate the confocal volume of the setup for $621 \mathrm{~nm}$ excitation using a $90 \mathrm{~nm}$ pinhole; a diffusion time of $42 \mu \mathrm{s}$ and a structural parameter $\left(\omega_{z} / \omega_{x y}\right)$ between 5 and 8 were obtained, resulting in a confocal volume of approximately $0.4 \mathrm{fL}$. Rhodamine $110\left(\mathrm{D}=4.3 \times 10^{-10} \mathrm{~m}^{2} \mathrm{~s}^{-1}\right.$ in water at $\left.20^{\circ} \mathrm{C}\right)$ was used to calibrate the confocal volume of the setup for $488 \mathrm{~nm}$ excitation using a $75 \mu \mathrm{m}$ pinhole; a diffusion time of $19 \mu \mathrm{s}$ and a structural parameter between 6 and 8 were obtained, resulting in a confocal volume of approximately $0.2 \mathrm{fL}$

FFS data processor version 2.3 (Scientific Software Technologies Software Centre, Minsk, Belarus) was used to fit the data to a diffusion model including triplet state. The parameter fitting was based on the Marquardt-Levenberg nonlinear method of least squares. The confidence intervals of the recovered parameters were calculated by the exhaustive search method. Those procedures were all performed within the software package, more details have been described by Skakun et al. (2014).

\subsection{Gel composition analysis by size-exclusion chromatography}

Digestion of WPI gels was performed inside the Teflon tubes (inner diameter $2 \mathrm{~cm}$, length $10 \mathrm{~cm}$ ) to impose one-dimensional diffusion (assuming the radial variations are negligible) and digestion: a small upper part of the prepared gel was removed and $2 \mathrm{~mL}$ SGF was added on top of the remaining gel. The gels were then digested in a $37^{\circ} \mathrm{C}$ water bath for $6 \mathrm{~h}$. Afterwards, the SGF was removed and pepsin in SGF was inactivated in a pre-heated Eppendorf Thermomixer Comfort (Eppendorf AG, Hamburg, Germany) at $90{ }^{\circ} \mathrm{C}$, shaking at $1400 \mathrm{rpm}$ for $5 \mathrm{~min}$. The WPI gels were cut perpendicularly to the axis of the tube ( $2 \mathrm{~mm}$ thick slices). In some experiments, the slices were crushed and soaked overnight in water $(0.5 \mathrm{wt} \%$ 
dry matter of gel in water including pepstatin $\left(30 \mu \mathrm{molL}^{-1}\right)$ to inhibit pepsin activity) for analyzing the water-soluble content inside the gel; others were immediately frozen and lyophilized for analyzing the gel composition.

The lyophilized gel slices were ground with a mortar and dissolved overnight in a solvent consisting of $8 \mathrm{~mol} / \mathrm{L}$ urea and $0.03 \mathrm{~mol} / \mathrm{L}$ dithiothreitol (DTT) (0.5 wt \% gel dry matter in solvent). The dissolved gels were then analyzed by size-exclusion chromatography with a combination of a TSKgel G2000SWxl and TSKgel G3000SWxl column (Tosoh Bioscience LLC, King of Prussia, PA, USA), using a method as described previously (Luo et al., 2015) except that the standard proteins were also dissolved in the same urea and DTT solution. The gel composition analysis was performed in duplicate. The water-soluble content was also analyzed using size-exclusion chromatography.

\subsection{Scanning electron microscopy}

WPI gels were cut into $7 \mathrm{~mm} \times 5 \mathrm{~mm}$ diameter cylindrical samples and digested in SGF for $1 \mathrm{~h}$ in a static soaking system (Luo et al., 2015). The samples were fixated with $3 \%(\mathrm{v} / \mathrm{v})$ glutaraldehyde immediately for at least $2 \mathrm{~h}$ and subsequently rinsed with Milli-Q water ( $310 \mathrm{~min}$ ). The samples were then immersed in $40 \mathrm{mmol} \mathrm{L}^{-1}$ osmium tetroxide for $2 \mathrm{~h}$ to $4 \mathrm{~h}$, rinsed with Milli-Q water ( $\left.3 \times 10 \mathrm{~min}\right)$ and dehydrated in an ethanol-water gradient series $(10 \%, 30 \%, 50 \%, 70 \%, 90 \%, 100 \%$ volume fraction for 15 min each). Afterward, the samples were dried in a critical point dryer (CPD 030, Bal-Tec AG, Balzers, Liechtenstein) with supercritical $\mathrm{CO}_{2}$. The dried samples were glued on stubs using conductive carbon glue and coated with $12 \mathrm{~nm}$ tungsten by a Leica EM MED020 sputter coater (Leica Microsystems, Wetzlar, Germany). The microstructures of samples were observed using a Magellan 400 field emission scanning electron microscope (FEI, Hillsboro, OR, USA) at room temperature with $2 \mathrm{kV}$ voltage.

\section{Results and discussion}

\subsection{Diffusivity of enhanced green fluorescent protein and pepsin}

The diffusion coefficients of fluorescently labeled pepsin as well as EGFP were determined using FCS. The autocorrelation curves obtained for EGFP in water and WPI gels are reported in Fig. 1A. The typical autocorrelation curves were fitted with a one-component model resulting in an optimal fit indicated by the straightness of the weighted residuals and low chi square values.

The autocorrelation curves for labeled pepsin in water and WPI gels are displayed in Fig. 1B. Data analysis [eq. (2)] revealed different species with distinct mobility. The pepsin conjugate (PepA633) curve in water (red circles) shows a two-component behavior $[\mathrm{n}=2$ in eq. (2)]. The faster component has similar diffusion time as free Alexa 633, which suggests the presence of free Alexa 633 molecules: we hypothesized that Pepsin-Alexa 633 conjugation is not completely stable and part of the Alexa 633 is released slowly 

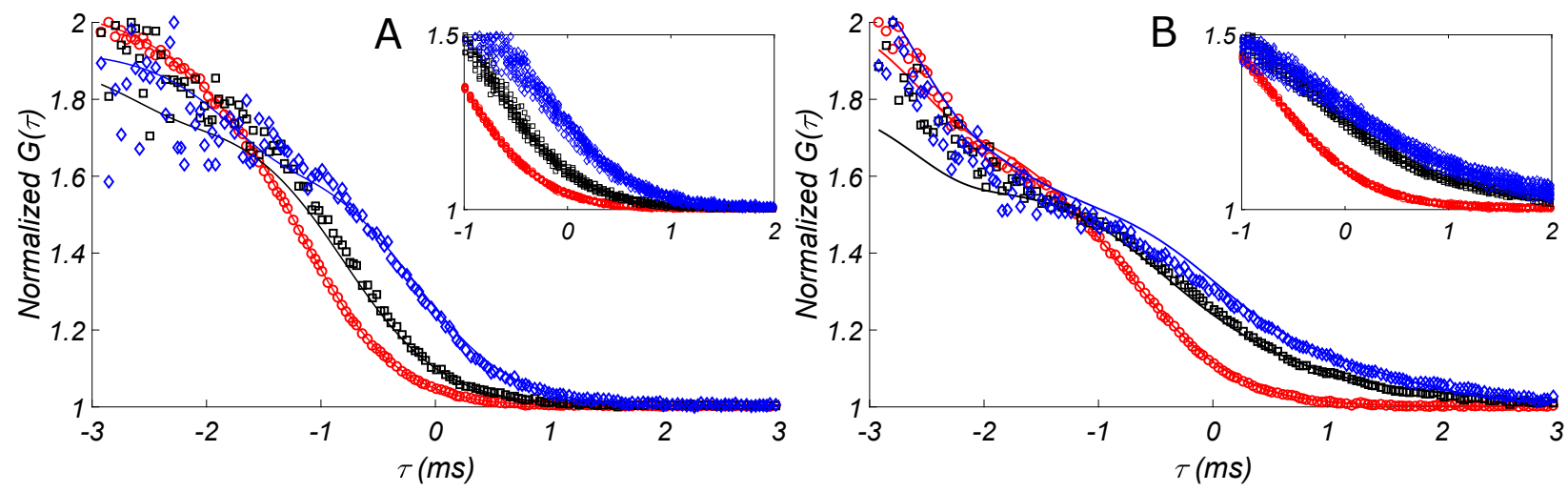

Figure 1: Examples of FCS autocorrelation curves. We show one measurement each for EGFP (A) and Pepsin (B) in water (red circle), in whey protein isolate (WPI) gel of $15 \mathrm{wt} \%$ (black square) and of $20 \mathrm{wt} \%$ (blue diamond). The corresponding lines are the fitted curves of diffusion models based on 10 measurements in one experiment. Those experiments of 10 measurements are shown in the insets.

from the conjugate over time, although the conjugate was quite pure after purification and was stored in $20 \%$ glycerol at $-25^{\circ} \mathrm{C}$. Therefore, we set the diffusion time of the faster component as equal to that of free Alexa 633 and regarded the other component as the PepA633 conjugate.

In contrast, the curves of the PepA633 conjugate in both types of the WPI gels have higher variance (see the black squares and blue diamonds in the inset of Fig. 1B) than those for EGFP in WPI gels and PepA633 in water. A three-component model was needed to describe the autocorrelation curve $[\mathrm{n}=3$ in eq. (2)]. Among the three components yielded, the diffusion coefficient of the fastest component is in the range of free Alexa 633 with an amplitude of approximately $40 \%$. The amplitude of the second component is around $50 \%$, and its diffusion time was of the same order of magnitude as the diffusivity of PepA633 in water. The third component is around $10 \%$, with a diffusion time of 80 ms regardless of the percentage of WPI gel type. This diffusion time corresponds to an extremely small diffusion coefficient of $2 \times 10^{-13} \mathrm{~m}^{2} \mathrm{~s}^{-1}$. If we exclude the fraction of free Alexa 633 , this slow diffusion represents $17 \%$ of the total amplitude.

The slowest mode of diffusion may have several causes:

1. The experiments were performed under neutral $\mathrm{pH}$, where PepA633, EGFP and the whey protein gel network are all negatively charged. Both electrostatic attraction and repulsion can hinder diffusion of charged particles (Stylianopoulos et al., 2010; Thorne et al., 2008). However, since EGFP did not show the slow diffusion mode, this cannot explain why the slow diffusion mode was found only with the labeled pepsin.

2. Aggregation of fluorescent conjugates may also cause a slower diffusion mode, but there was no evidence for slow diffusion mode of PepA633 in water. In addition, a diffusion coefficient that is 100 $\mathrm{x}$ lower would require massive aggregates of 106 molecules (assuming Stokes-Einsteins equation to be valid inside the WPI matrix), which would have been visible during the experiments. 


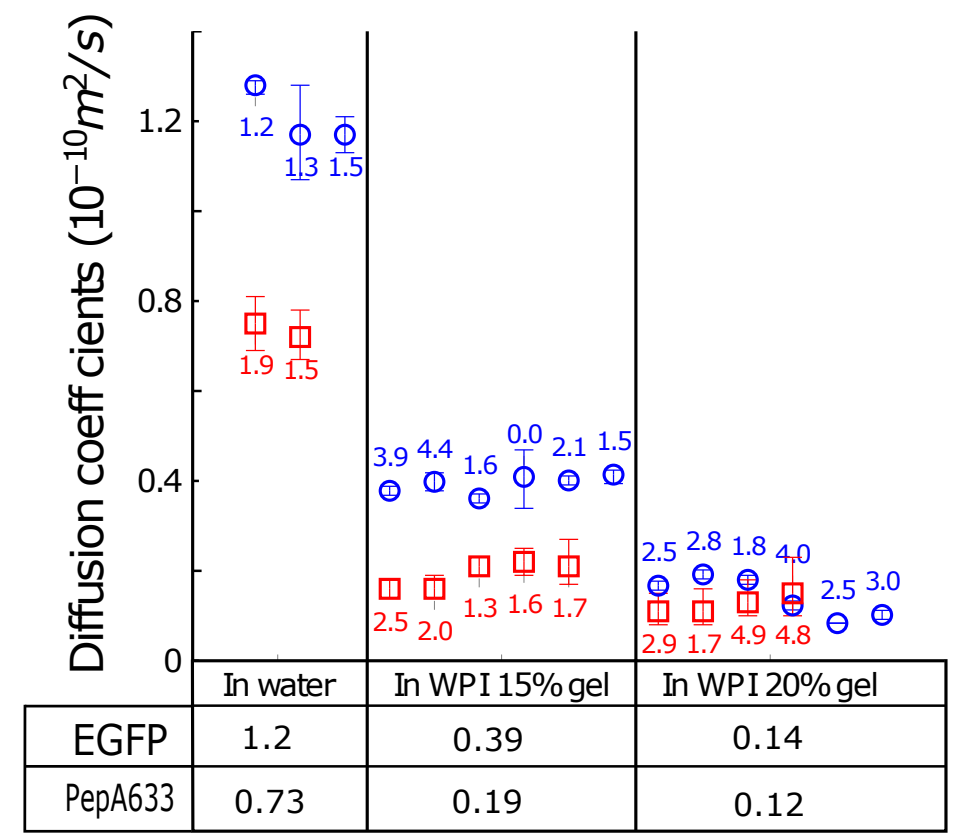

Figure 2: Diffusion coefficients of Enhanced Green Fluorescent Protein (EGFP, blue circle) and Pepsin-Alexa 633 conjugates (PepA633, red square) in water and in Whey Protein Isolate (WPI) gel $15 \mathrm{wt} \%$ and $20 \mathrm{wt} \%$. Each data point is calculated from the fitted parameter of 10 measurements in one experiments; the number next to it is the $\chi^{2}$ value from the nonlinear least square fitting process; error bars are its $95 \%$ confidence intervals. The values in the table below are the mean values of the correspondent repeated experiments.

3. A bimodal pore size distribution could explain a fast and a slow diffusion mode; however, in this case, one would expect that it would be seen with both proteins, EFGP and the PepA633 conjugate.

4. In FCS studies, detecting extra species with slow diffusion could indicate probe-network interaction (Wöll, 2013). The slow diffusion mode occurs only with the conjugate, and the most obvious difference between the two proteins is that pepsin naturally binds to proteins. Our hypothesis, therefore, is that the slow conjugate diffusion mode is related to the pepsin that forms a temporary complex with the WPI matrix. The fact that the fraction of conjugate in the slow mode is the same in both gels supports this hypothesis.

The diffusion coefficients are reported in Fig.2; we regard the second component as the PepA633 diffusing in WPI gels. The EGFP diffusion in water is $1.2 \times 10^{-10} \mathrm{~m}^{2} \mathrm{~s}^{-1}$, which is in agreement with other studies (Nolles et al., 2015). The diffusion coefficient of PepA633 in water is $7.3 \times 10^{-11} \mathrm{~m}^{2} \mathrm{~s}^{-1}$, which is slightly lower than the pepsin diffusion coefficient in water as reported previously $\left(8.7 \times 10^{-11} \mathrm{~m}^{2} \mathrm{~s}^{-1}\right.$; (Tyn \& Gusek, 1990)). The larger diffusion coefficient of EGFP in water compared with that of PepA633 may stem from its lower molecular mass and thus smaller hydrodynamic radius (Erickson (2009) demonstrated the correlation between molecular weight and hydrodynamic radius of proteins). In 15\% WPI gel, the diffusion coefficients of EGFP and PepA633 are both smaller than in water, while the EGFP diffusion remains faster than that of PepA633. In 20\% WPI gel, the differences between EGFP and PepA633 are negligible. 
The hindered diffusion of solute in hydrogels has been explained using various theories. For instance, the free volume theory suggested that hydrogels reduce free volume that allows solute to diffuse; the hydrodynamic theory proposed that hydrogels enhance the frictional drag on the solute (Amsden, 1998). Thus, as shown in our experiments, protein gels with higher protein fraction have greater hindrance on the PepA633 and EGFP diffusion. The diffusion coefficients can be used to estimate the enzyme concentration gradient inside protein gels during digestion, using Ficks second law [eq. 4] and its integration for one-dimensional diffusion [eq. 5] (Walstra, 2002):

$$
\begin{gathered}
\frac{\partial C}{\partial t}=D \frac{\partial^{2} C}{\partial x^{2}} \\
C=C_{0}\left(1-\frac{2}{\sqrt{\pi}} \int_{0}^{\frac{x}{2 \sqrt{D t}}} e^{-t^{2}} d t\right)
\end{gathered}
$$

where $C$ is the concentration dependent on the position $(\mathrm{x})$ and time $(\mathrm{t}), C_{0}$ is the constant concentration at the boundary; $\mathrm{D}$ is the diffusion coefficient. Fig. 3 shows the relative pepsin concentration $\left(C / C_{0}\right)$ along the diffusion distance $(\mathrm{x}=0 \mathrm{~mm}$ to $2 \mathrm{~mm})$ in one dimension after a certain period of time $(1 \mathrm{~h}$ or $6 \mathrm{~h})$, assuming that the bulk concentration of pepsin and diffusion coefficients are constant regardless of time and location. One can either only consider the fast mode of pepsin diffusion or include the slow mode diffusion that $17 \%$ of the pepsin exhibits, as indicated above. The dashed lines in Fig. 3 excluded the slow mode diffusion, and the solid lines included the slow mode diffusion.

Within $6 \mathrm{~h}$, which is in the range of gastric emptying time of solid meals (Bornhorst et al., 2013; Collins et al., 1991), the pepsin concentration reaches a significant level only in the first $2 \mathrm{~mm}$ of the gels. Thus, the enzymatic hydrolysis of gel matrix is constrained within a thin layer. This may cause the limited digestion of protein gel structure compared with protein in solution that was found earlier (Luo et al., 2015). However, the differences in the pepsin concentration between $15 \%$ and $20 \%$ WPI gels are much smaller than the differences in the degrees of hydrolysis obtained in our previous research, where WPI 15\% gel underwent a much higher degree of hydrolysis than WPI 20\% gel during simulated gastric digestion (Luo et al., 2015). Therefore, the diffusional constraint might not be the only explanation for the hindered digestion of protein in gel matrices. The FCS experiments were performed at neutral $\mathrm{pH}$, where pepsin has little activity; while in realistic digestion, one should consider that the action of pepsin may change the gel structure and consequently change the pepsin diffusivity.

\subsection{Gel composition analysis by size-exclusion chromatography}

To validate and explore the effect of pepsin diffusion in protein gel digestion, we dissolved the gel slices with urea and DTT after digestion, and analyzed the gel composition using size-exclusion chromatography. The resulting chromatograms for WPI gels of $15 \mathrm{wt} \%$ and $20 \mathrm{wt} \%$ are shown in Fig. 4 . The compositions 

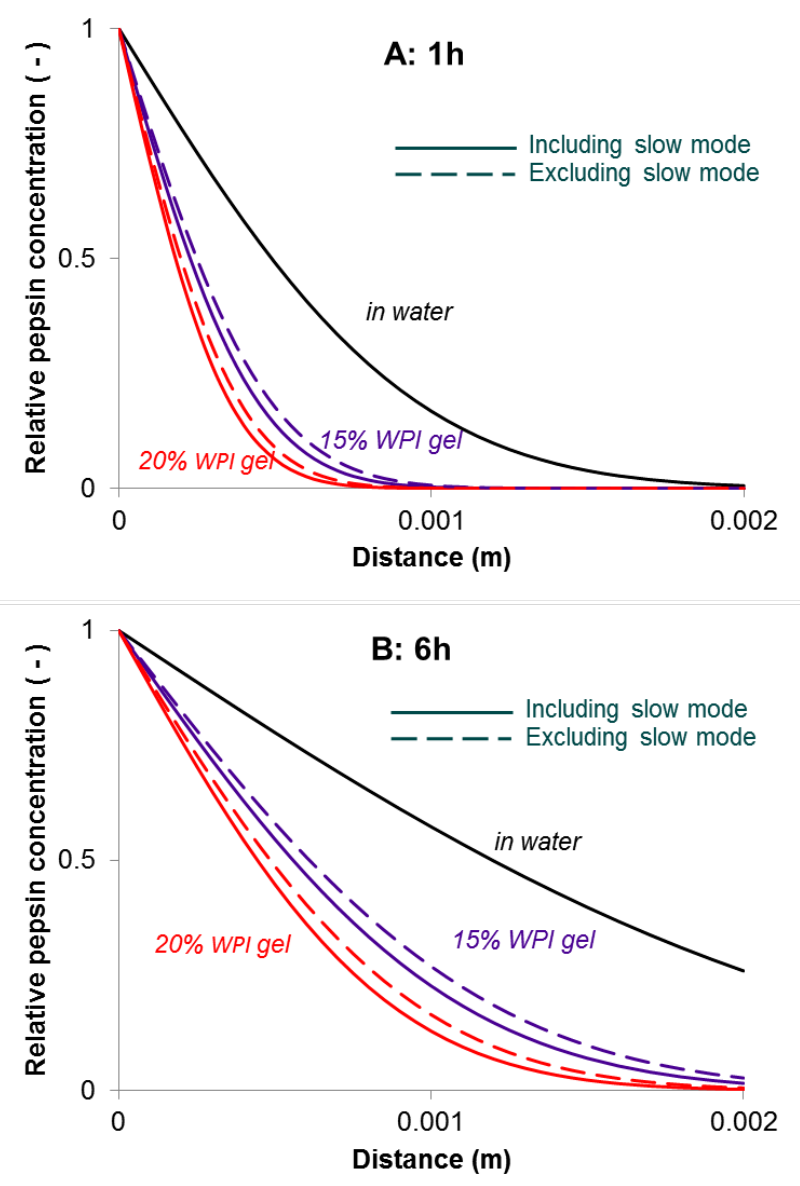

Figure 3: Size-exclusion chromatograms of composition analysis of whey protein isolate gels $15 \mathrm{wt} \%$ (A) and $20 \mathrm{wt} \%$ (B) after $6 \mathrm{~h}$ digestion and the correspondent simulated gastric fluid (SGF). Each gel slice has a thickness of $2 \mathrm{~mm}$.
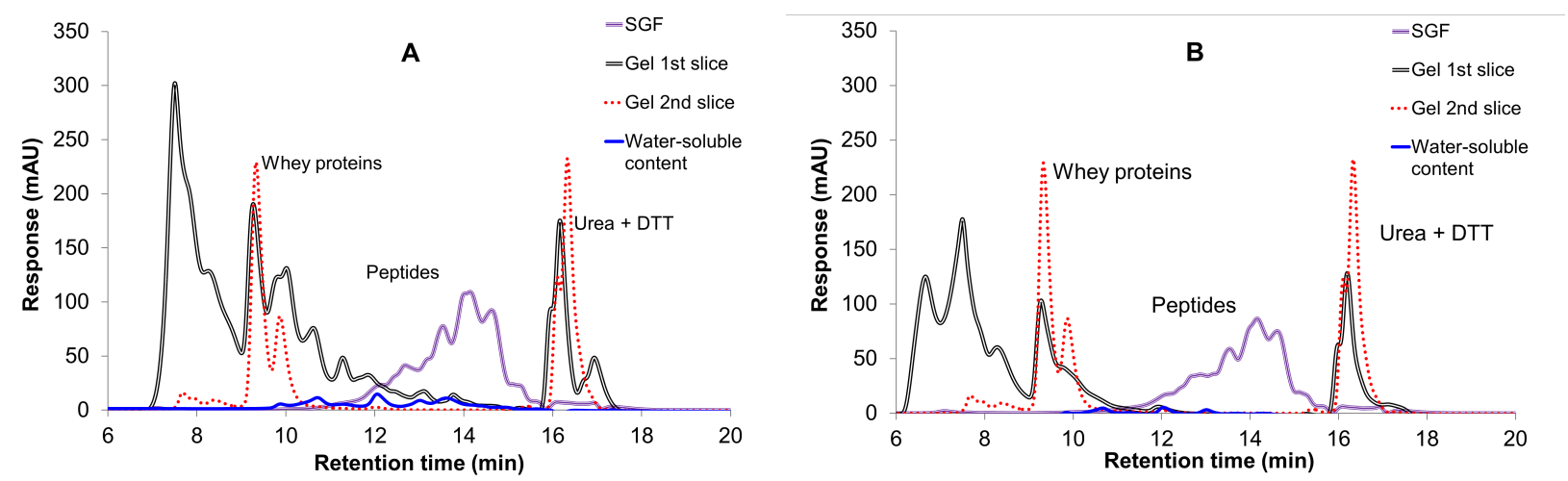

Figure 4: Size-exclusion chromatograms of composition analysis of whey protein isolate gels $15 \mathrm{wt} \%$ (A) and $20 \mathrm{wt} \%$ (B) after $6 \mathrm{~h}$ digestion and the correspondent simulated gastric fluid (SGF). Each gel slice has a thickness of $2 \mathrm{~mm}$. 


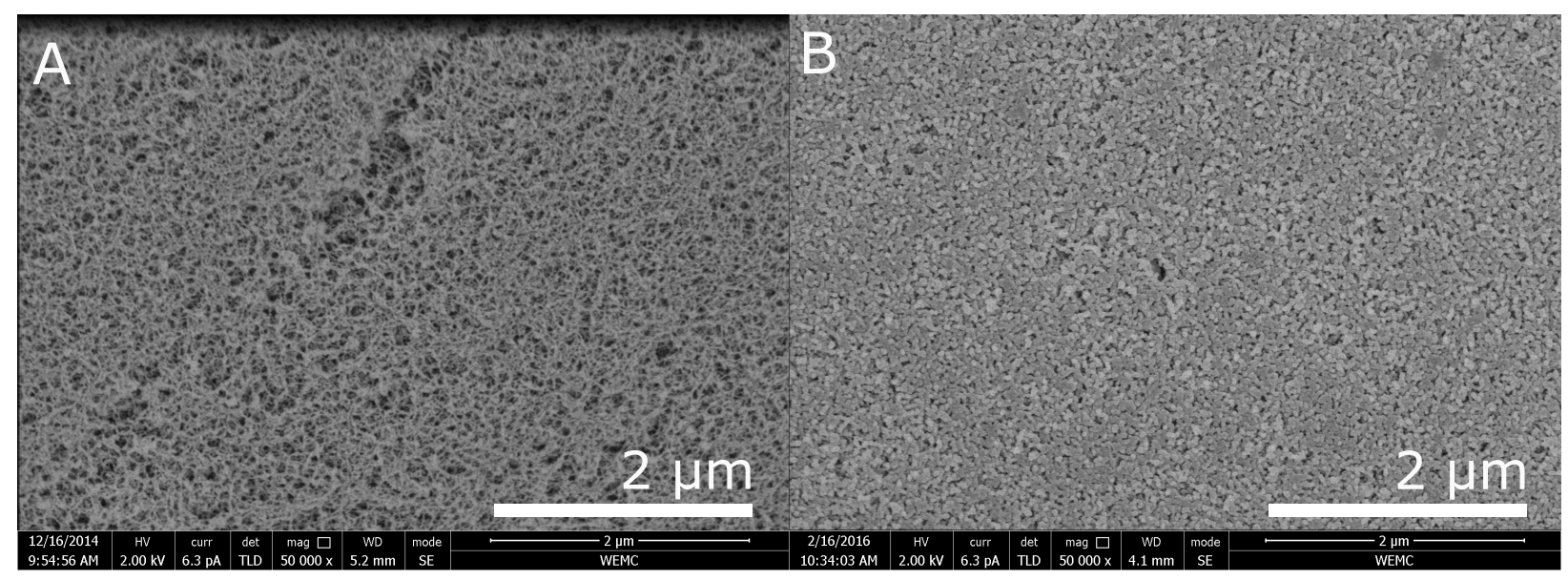

Figure 5: Scanning electron micrograph of the original surface of whey protein isolate gels $15 \mathrm{wt} \%$ (A) and $20 \mathrm{wt} \%$ (B).

of the second and onward slices were identical to that of undigested gels for both gels (only the second slices were shown), while the first slice was distinctively different. This shows that indeed the pepsin did not affect the second slices. The chromatogram of the first slice of the WPI gel showed several peaks after $10 \mathrm{~min}$ of retention time, which indicates the presence of hydrolytic products. Therefore, hydrolysis had occurred in and only within the first $2 \mathrm{~mm}$ of the gel, which is in accordance with the estimation based on enzyme diffusion. Moreover, the hydrolysis fragments in the first gel slice were larger than the small peptides in the SGF, based on their distributions in retention time. The 20\% WPI gel (Fig. 4B) showed the same trend as the $15 \%$ WPI gel, but with lower amounts of peptides in the gel slice and in the SGF.

We hypothesized previously that the different peptide distributions obtained during the digestion of a whey protein solution and of a gel (Luo et al., 2015) may be caused for two reasons: the hydrolysis fragments may either be still attached to the WPI network, or they may be constrained in their egression. The water-soluble content inside the WPI gels first slices showed similar amount of small peptides (retention time $>12 \mathrm{~min}$ ) but a lower amount of larger peptides (retention time $<12 \mathrm{~min}$ ), compared with that of the dissolved first slices of gels (Fig. 4). Therefore, those larger peptides may be still attached to the WPI gel network and can only be dissolved with urea and DTT, which supports the first hypothesis.

Large peaks at $7.5 \mathrm{~min}$ retention time were observed in the first slices that were digested either with SGF or with $\mathrm{pH} 2$ acidic water (data not shown). We presume that is the stable self-assembly of whey protein formed under acidic condition, since it has been reported previously that -lactoglobulin at $\mathrm{pH} 2$ can form irreversible fibrillar protein aggregates (Akkermans et al., 2008).

\subsection{Microstructure of WPI gels}

Scanning electron microscopy was employed to observe the effect of pepsin diffusion on the microstructure of WPI gels. The surfaces of original WPI $15 \mathrm{wt} \%$ and $20 \mathrm{wt} \%$ gels, shown in Fig. 3.5, are fine-stranded 
gels with rather homogeneous microstructure and a fine network. The network of the $20 \mathrm{wt} \%$ gel has smaller pore size than the $15 \%$ gel network, in agreement with the lower diffusion rates of both EGFP and PepA633 in the $20 \%$ gel. We found that the diffusivity of PepA633 was reduced by about $70 \%$ in $15 \mathrm{wt} \%$ gel and $80 \%$ in $20 \mathrm{wt} \%$ gel compared with its diffusivity in water (Fig. 2). Colsenet et al. (Colsenet, Söderman \& Mariette, 2006a) reported on the diffusivity of poly(ethylene glycol)s in whey protein gels; they found a reduction of $70 \%$ for the $82 \mathrm{kDa}$ poly(ethylene glycol) in a $19.35 \mathrm{wt} \%$ WPI gel (heated at $70{ }^{\circ} \mathrm{C}$ for $30 \mathrm{~min}$, $\mathrm{NaCl} 0.1 \mathrm{mmol} \mathrm{L}^{-1}$ ) compared with $D_{0}$ in water, which is in the same order of magnitude for both EGFP and pepsin in $20 \mathrm{wt} \%$ WPI gel. This small discrepancy in diffusivity reduction may stem from the different gel microstructures caused by the differences in heat treatment and ionic strength: a higher ionic strength during gel formation leads to a particulate gel structure and a larger pore sizes (Doi, 1993). Moreover, poly(ethylene glycol)s are linear, flexible molecules that can adjust their shape according to the network (Petit et al., 1996), while EGFP and PepA633, which are globular, rigid and charged proteins, thus behave different than poly(ethylene glycol)s.

After $1 \mathrm{~h}$ of digestion, both the 15\% and 20\% WPI gel surfaces changed compared with native samples and some heterogeneity appeared (Fig. 6): some areas appeared to be smoother than others; the smooth areas remained rather homogeneous, while the structure became more open than the original microstructure. We suppose that the pepsin hydrolysis weakens the gel microstructure. The rougher areas resemble fragments with more random formation, which may be aggregates induced by hydrolysis and the acidity. We remind the reader that stable aggregates were also observed in the size-exclusion chromatograms (Fig. 4).

The loosened microstructure in the smooth area may lead to faster pepsin diffusion.. Thus, the pepsin concentration in realistic gastric digestion may be slightly higher than our estimation in Chapter Section 3.1 where we assumed the diffusion coefficients are constant regardless of time and location. Regarding the different digestion between $15 \%$ and $20 \%$ WPI gels, the diffusional constraint might not be the only hindrance; with the microstructure observation we hypothesize that the different rate of structure loosening may be another cause. The summed effect of different diffusion limitations, hydrolysis rates and differences in microstructure and mechanical strength may have caused the difference in disintegration and digestion of two WPI gels.

\section{Conclusion}

We measured the diffusivity of pepsin in whey protein gels by FCS and found significantly slower pepsin diffusion in $15 \mathrm{wt} \%$ and $20 \mathrm{wt} \%$ WPI gels compared with pepsin diffusion in water, due to steric hindrance in the gel microstructure. The diffusivity of EGFP in the respective protein gels was similar to that of pepsin, but did not show a characteristic slow diffusion mode that was observed for pepsin. This 


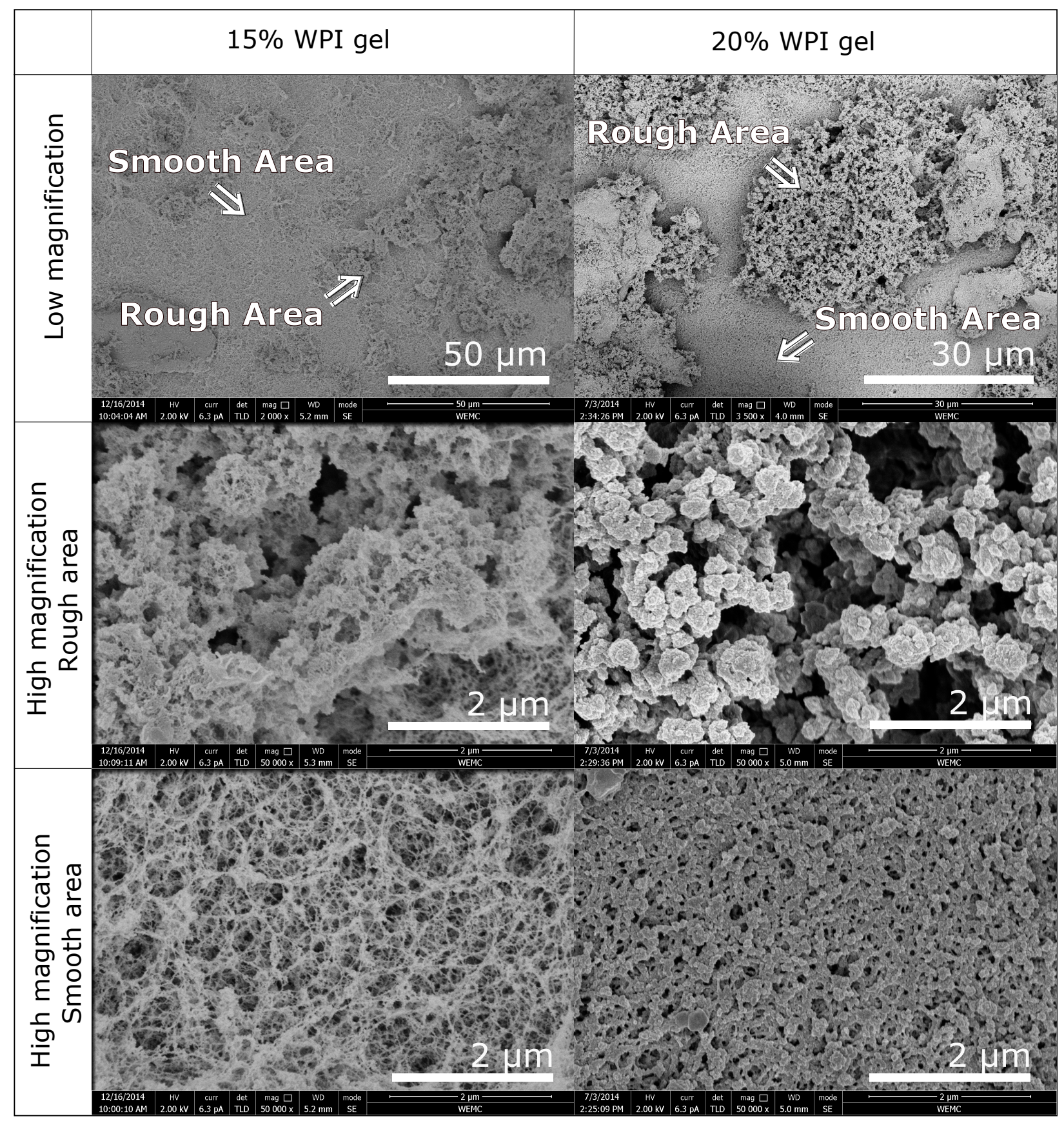

Figure 6: Scanning electron micrograph of whey protein isolate (WPI) gel (15 wt $\%$ and $20 \mathrm{wt} \%)$ surface after $1 \mathrm{~h}$ static soaking in simulated gastric fluid. Low magnification images show the overview and high magnification images show the details of rough area and smooth area. 
slow mode, for about $17 \%$ of the pepsin, is thought to be the pepsin that is temporarily bound to the WPI protein matrix.

Pepsin hydrolysis in the gel matrix is constrained to a thin layer at the surface for timescales that are similar to human gastric digestion. Since pepsin has limited penetration depth, digestion processes are constrained largely to the surface area of the gel particles and on the surface erosion rate.

Analysis of hydrolysis products inside the gels and in SGF indicated that also the reaction products cannot diffuse freely out of the gel but are significantly retained inside the gel structure.

By combining diffusivity study, gel composition analysis and microstructure observation, we can conclude that the mode of WPI gel digestion is determined by the summed effect of diffusion limitation, hydrolysis rate and microstructure transformation. This summed effect could also have caused the distinctive digestion rate between WPI $15 \mathrm{wt} \%$ and $20 \mathrm{wt} \%$ gels.

We demonstrated the digestion process of WPI gels from a mechanistic view at the microscale level. This knowledge may be used further to predict the digestion of solid food in general, from protein-based food to more complex food structures.

\section{Acknowledgment}

The authors thank Marcel Giesbers of Wageningen Electron Microscopy Centre for the scanning electron microscopy work; and also thank Olga Nikoloudaki, Doan Thi Thanh Thao, Jolieke Kerckhoffs and Remco de Lange for part of the experimental work.

\section{References}

Akkermans, C., van der Goot, A. J., Venema, P., van der Linden, E., \& Boom, R. M. (2008). Formation of fibrillar whey protein aggregates: Influence of heat and shear treatment, and resulting rheology. Food Hydrocolloids, 22, 1315-1325. doi:10. 1016/ j . foodhyd.2007.07.001.

Amsden, B. (1998). Solute Diffusion within Hydrogels. Mechanisms and Models. Macromolecules, 31, 8382-8395. doi:10. 1021/ ma980765f.

Bornhorst, G. M., Chang, L. Q., Rutherfurd, S. M., Moughan, P. J., \& Singh, R. (2013). Gastric emptying rate and chyme characteristics for cooked brown and white rice meals in vivo. Journal of the Science of Food and Agriculture, . doi:10.1002/ jsfa.6160.

Bornhorst, G. M., \& Singh, R. P. (2014). Gastric Digestion In Vivo and In Vitro: How the Structural Aspects of Food Influence the Digestion Process. Annual Review of Food Science and Technology, 5, 111-132. doi:10. 1146/annurev-food-030713-092346.

Collins, P. J., Houghton, L. A., Read, N. W., Horowitz, M., Chatterton, B. E., Heddle, R., \& Dent, J. (1991). Role of the proximal and distal stomach in mixed solid and liquid meal emptying. Gut, 32, 615-619. doi:10.1136/gut.32.6.615.

Colsenet, R., Söderman, O., \& Mariette, F. (2006a). Effect of casein concentration in suspensions and gels on poly (ethylene glycol) s NMR self-diffusion measurements. Macromolecules, 38, 9171-9179.

Colsenet, R., Söderman, O., \& Mariette, F. (2006b). Pulsed Field Gradient NMR Study of Poly(ethylene glycol) Diffusion in Whey Protein Solutions and Gels. Macromolecules, 39, 1053-1059. doi:10 . 1021/ma0519922. 
Croguennoc, P., Nicolai, T., Kuil, M. E., \& Hollander, J. G. (2001). Self-diffusion of Native Proteins and Dextran in Heat-set Globular Protein Gels. The Journal of Physical Chemistry B, 105, 5782-5788. doi:10. 1021/ jp0043726.

Doi, E. (1993). Gels and gelling of globular proteins. Trends in Food Science E Technology, 4, 1-5. doi:10.1016/S0924-2244(05) 80003-2.

Erickson, H. P. (2009). Size and Shape of Protein Molecules at the Nanometer Level Determined by Sedimentation, Gel Filtration, and Electron Microscopy. Biological Procedures Online, 11, 32-51. doi:10. 1007/s12575-009-9008-x.

Fersht, A. (1985). Structures and mechanisms of selected enzymes. In Enzyme Structure and Mechanism (pp. 389-452). New York: W. H. Freeman \& Co. (2nd ed.).

Guo, Q., Ye, A., Lad, M., Ferrua, M., Dalgleish, D., \& Singh, H. (2015). Disintegration kinetics of food gels during gastric digestion and its role on gastric emptying: An in vitro analysis. Food E Function, 6, 756-764. doi:10.1039/C4F000700J.

Inouye, K., \& Fruton, J. S. (1967). Studies on the Specificity of Pepsin*. Biochemistry, 6, 1765-1777.

Kwaaitaal, M., Schor, M., Hink, M. A., Visser, A. J., \& de Vries, S. C. (2011). Fluorescence Correlation Spectroscopy and Fluorescence Recovery After Photobleaching to Study Receptor Kinase Mobility In Planta. In N. Dissmeyer, \& A. Schnittger (Eds.), Plant Kinases number 779 in Methods in Molecular Biology (pp. 225-242). New York: Humana Press. doi:10. 1007/978-1-61779-264-9_13.

Lakowicz, J. R. (2006). Fluorescence Correlation Spectroscopy. In Principles of Fluorescence Spectroscopy (pp. 797-840). New York: Springer US.

Luo, Q., Boom, R. M., \& Janssen, A. E. M. (2015). Digestion of protein and protein gels in simulated gastric environment. LWT - Food Science and Technology, 63, 161-168. doi:10.1016/ j . lwt.2015.03.087.

Nolles, A., Westphal, A. H., de Hoop, J. A., Fokkink, R. G., Kleijn, J. M., van Berkel, W. J. H., \& Borst, J. W. (2015). Encapsulation of GFP in Complex Coacervate Core Micelles. Biomacromolecules, 16, 1542-1549. doi:10.1021/acs.biomac .5b00092.

Nyemb-Diop, K., Causeur, D., Jardin, J., Briard-Bion, V., Guérin-Dubiard, C., Rutherfurd, S. M., Dupont, D., \& Nau, F. (2016). Investigating the impact of egg white gel structure on peptide kinetics profile during in vitro digestion. Food Research International, 88, 302-309. doi:10.1016/ j foodres.2016.01.004.

Petit, J.-M., Zhu, X. X., \& Macdonald, P. M. (1996). Solute Probe Diffusion in Aqueous Solutions of Poly(vinyl alcohol) As Studied by Pulsed-Gradient Spin-Echo NMR Spectroscopy. Macromolecules, 29, 70-76. doi:10. 1021/ma9510597.

Piper, D., \& Fenton, B. H. (1965). pH stability and activity curves of pepsin with special reference to their clinical importance. Gut, 6, 506-508.

Royant, A., \& Noirclerc-Savoye, M. (2011). Stabilizing role of glutamic acid 222 in the structure of Enhanced Green Fluorescent Protein. Journal of Structural Biology, 174, 385-390. doi:10.1016/ j . j sb .2011.02.004.

Singh, H., Ye, A., \& Ferrua, M. J. (2015). Aspects of food structures in the digestive tract. Current Opinion in Food Science, 3, 85-93. doi: $10.1016 /$ j.cofs.2015.06.007.

Skakun, V., Digris, A., \& Apanasovich, V. (2014). Global Analysis of Autocorrelation Functions and Photon Counting Distributions in Fluorescence Fluctuation Spectroscopy. In Y. Engelborghs, \& A. J. W. G. Visser (Eds.), Fluorescence Spectroscopy and Microscopy number 1076 in Methods in Molecular Biology (pp. 719-741). New York: Humana Press.

Stylianopoulos, T., Poh, M.-Z., Insin, N., Bawendi, M. G., Fukumura, D., Munn, L. L., \& Jain, R. K. (2010). Diffusion of Particles in the Extracellular Matrix: The Effect of Repulsive Electrostatic Interactions. Biophysical Journal, 99, 1342-1349. doi:10. 1016/ j . bp j . 2010.06.016.

Thorne, R. G., Lakkaraju, A., Rodriguez-Boulan, E., \& Nicholson, C. (2008). In vivo diffusion of lactoferrin in brain extracellular space is regulated by interactions with heparan sulfate. Proceedings of the National Academy of Sciences, 105, 8416-8421. doi:10. $1073 /$ pnas. 0711345105.

Tyn, M. T., \& Gusek, T. W. (1990). Prediction of diffusion coefficients of proteins. Biotechnology and Bioengineering, 35, 327-338. doi: $10.1002 /$ bit. 260350402.

Videcoq, P., Steenkeste, K., Bonnin, E., \& Garnier, C. (2013). A multi-scale study of enzyme diffusion in macromolecular solutions 
and physical gels of pectin polysaccharides. Soft Matter, 9, 5110-5118. doi:10. 1039/C3SM00058C.

Walstra, P. (2002). Physical Chemistry of Foods. Boca Raton, Florida: CRC Press.

Whitney, E. N., Cataldo, C. B., \& Sharon, R. R. (1998). Understanding Normal and Clinical Nutrition. (5th ed.). Wadsworth Publishing Company, Inc.

Wöll, D. (2013). Fluorescence correlation spectroscopy in polymer science. RSC Advances, 4, 2447-2465. doi:10. 1039/C3RA44909B. 